\title{
LA PRIMERA EXPERIENCIA DE PRACTICUM: UN ESTUDIO EXPLORATORIO CON ALUMNADO Y PROFESORADO-TUTOR DEL GRADO DE EDUCACIÓN INFANTIL
}

\author{
Pilar Gil Molina \\ Universidad del País Vasco (UPV-EHU)
}

\begin{abstract}
RESUMEN: Numerosos estudios afirman que el período de prácticas es clave en la formación del alumnado porque le proporciona la oportunidad de trabajar y desarrollar competencias profesionales (Cid, Pérez y Sarmiento, 2011; Novella, 2011; Rodríguez-Gómez, Armengol y Meneses, 2017; Rosales, 2013; Tejada y Ruiz, 2013; Zabalza, 2011). Sin embargo, ¿cuál es la percepción del alumnado y del profesorado-tutor de los centros escolares? ¿Cómo valoran esta experiencia de prácticas? ¿En base a qué criterios realizan esas valoraciones? En este trabajo se ha intentado dar respuesta a estas cuestiones recogiendo la opinión de un grupo de alumnas del Grado de Maestro en Educación Infantil de la Universidad del País Vasco (UPV/EHU) del campus de Gipuzkoa y contrastándolas con las valoraciones realizadas por el profesorado-tutor de los centros escolares en los que realizaron dichas prácticas. Los resultados indican que ambos colectivos valoran positivamente esta primera experiencia de prácticas y dan especial importancia en estas prácticas iniciales a aspectos relacionados con la interacción personal, entre otros: la ayuda proporcionada por el alumnado, la iniciativa demostrada, la participación activa y la actitud colaborativa.
\end{abstract}

PALABRAS CLAVE: Practicum, formación inicial de maestros/as, tutores/as de prácticas, satisfacción del alumnado.

\section{ABOUT THE FIRST PRACTICUM EXPERIENCE: AN EXPLORATORY STUDY WITH UNIVERSITY STUDENTS AND TEACHER-TUTORS OF THE UNIVERSITY DEGREE IN CHILDHOOD EDUCATION}

\footnotetext{
ABSTRACT: The importance of Practicum as an essential component of teacher training education had been defended by several authors who affirmed that the internship period provides to students the opportunity to work and develop professional competences (Cid, Pérez y Sarmiento, 2011; Novella,
} 
2011; Rodríguez-Gómez, Armengol y Meneses, 2017; Rosales, 2013; Tejada y Ruiz, 2013; Zabalza, 2011). However, what do the students and the scholar teacher-tutors think about this formative period takes place? How do the students value his /her practical experience? What are the criteria that they value making these assessments? Trying to answer these questions, the assessments made by students of the University Degree in Childhood Education of the University of the Basque Country/Euskal Herriko Unibertsitatea (UPV/EHU) with their first experience of practices have been analyzed and contrasted with the assessments made by their teacher-tutor of the schools in which they performed these practices. The results indicate that both groups give a high rating to these first practices and base their assessments on elements related to personal interaction, such as: provided assistance, demonstrated initiative, active participation and collaborative attitude.

KEYWORDS: Practicum, preservice teacher education, student teacher supervisor, student satisfaction.

Recibido: 02/02/2018

Aceptado: 11/07/2018

Correspondencia: Pilar Gil Molina, Universidad del País Vasco, Plaza Oñati, 3, 20018 San Sebastián. Email: pilar.gil@ehu.eus.

\section{INTRODUCCIÓN}

La importancia del Practicum como componente esencial de la formación del profesorado es defendida por diversos autores que afirman que el período de prácticas proporciona al alumnado la oportunidad de trabajar y desarrollar competencias profesionales (Cid, Pérez y Sarmiento, 2011; García, 2006; González, 2011; Khortagen, Loughran y Russell, 2006; Novella, 2011; Ong'ondo y Jwan, 2009; RodríguezGómez, Armengol y Meneses, 2017; Rosales, 2013; Smith y Hodson, 2010; Tejada y Ruiz, 2013; Vick, 2006; Zabalza, 2011; Zeichner, 2010a, 2010b).

Estudios realizados con alumnado universitario de diferentes titulaciones (Cano, Orejudo y Cortés, 2012; Cid y Ocampo, 2007; García, Pascual y Fombona, 2011; González y Hevia, 2011; Melgarejo, Pantoja y Latorre, 2014; Pérez y Burguera, 2011; Pontes y Serrano, 2010; Sanjuán y Méndez, 2011; Tello, 2007) parecen constatar que éste valora de forma positiva y satisfactoria su experiencia de prácticas. Así mismo, los estudios realizados con el profesorado-tutor de los centros colaboradores de prácticas externas universitarias de diferentes áreas también coinciden en esta valoración acerca de la importancia formativa del Practicum (Liesa, Otal y Antonio, 2009; Manso y Martín, 2014; Martínez y Raposo, 2011a, 2011b; Sánchez, García y Ramírez, 2011) y destacan que una de las claves para el mejor funcionamiento de Practicum de los nuevos Grados de Maestro/a pasa por reforzar las estructuras de coordinación entre todos los agentes implicados (Elwes, Fernández y Rivas, 2012). 
Como en otras titulaciones universitarias, el Practicum de los planes de estudio de la Titulación del Grado de Maestro/a de Educación Infantil supone la inmersión del alumnado en los centros educativos con la finalidad de conocer la realidad educativa, integrar los conocimientos teórico-prácticos y adquirir competencias profesionales. En el plan de estudios del Grado de Educación Infantil de la Universidad del País Vasco (UPV/EHU) de Haur Hezkuntza (Educación Infantil en euskera) el Practicum es una asignatura troncal y obligatoria que se distribuye en tres asignaturas: Practicum I, Practicum II y Practicum III. Estas asignaturas se imparten, respectivamente, en el segundo, el tercero y el cuarto curso del plan de estudios. Cada una de las tres asignaturas tiene objetivos formativos diferenciados y supone para el alumnado una estancia en el centro escolar de duración e implicación creciente (ver Tabla 1):

- El Practicum I pretende proporcionar al alumnado un primer contacto con el centro escolar. El objetivo de estas prácticas iniciales es que el alumnado realice una observación activa y de carácter global, integrando los conocimientos teórico-prácticos aprendidos en el primer curso de la universidad con la realidad de la escuela y el entorno social de ésta, y dedicando una especial atención al tratamiento de género.

- El Practicum II pretende ayudar a que el alumnado, en un segundo contacto con la realidad escolar, realice una observación participativa y se implique en la realidad del aula, del ciclo y de la etapa. Por este motivo, se le solicita el diseño y el desarrollo de experiencias de enseñanza y aprendizaje a través de las cuales pueda poner en práctica los conocimientos adquiridos y reflexionar sobre su futuro desempeño docente.

- El Practicum III está relacionado con el minor (la mención o especialidad) del cuarto y último curso de Grado y supone un mayor nivel de conocimiento e implicación en la actividad del aula, del centro escolar y de la especialidad cursada. Por ejemplo, a través de su participación en los proyectos de innovación que se están realizando en el centro de prácticas, el alumnado ha de diseñar y desarrollar intervenciones educativas en áreas específicas como puede ser la atención a alumnado con necesidades educativas especiales.

Tabla 1. Prácticas externas curriculares del Grado de Maestro/a de Educación Infantil

\begin{tabular}{lclc}
\hline \multicolumn{1}{c}{ Asignatura } & Créditos & \multicolumn{1}{c}{ Estancia* } & Fechas \\
\hline Practicum I & 8 & 5 semanas & Octubre-noviembre \\
\hline Practicum II & 12 & 7 semanas & Febrero-abril \\
\hline Practicum III & 18 & 12 semanas & Noviembre-febrero \\
\hline *30 horas semanales en cada estancia & & \\
\hline
\end{tabular}

Estas prácticas curriculares se realizan en el marco de un convenio de colaboración suscrito por el Departamento de Educación, Política Lingüística y Cultura del Gobierno Vasco y la Universidad del País Vasco/Euskal Herriko Unibertsitatea (UPV/ EHU), están organizadas y coordinadas por el Vicedecanato de Practicum y la Comisión de Practicum del área de Magisterio de la Facultad de Educación, Filosofía 
y Antropología de dicha universidad. En ellas participan los tutores y las tutoras de prácticas (personal docente e investigador de diferentes departamentos universitarios) y los tutores y las tutoras de los centros escolares colaboradores, así como los coordinadores y las coordinadoras de prácticas que son responsables del Practicum de dichos centros escolares. Cada uno de estos agentes (ver Figura 1) asume unas funciones previamente establecidas:

- El coordinador o la coordinadora del centro escolar es la persona responsable de tutelar las actuaciones del alumnado de prácticas dentro del centro educativo y de mantener las relaciones correspondientes con la universidad. Es la persona que ha de facilitar la estancia del alumnado de prácticas en su centro, recibirle y acompañarle al inicio del proceso, nombrar al profesorado-tutor y concretar sus funciones en el proceso de formación del alumnado.

- El profesorado-tutor del centro ha de acoger, orientar, acompañar, proponer acciones, ayudar, supervisar, valorar al alumnado y comunicar al coordinador o a la coordinadora de su centro y al tutor o la tutora de la universidad las posibles incidencias que tengan lugar en el desarrollo de la estancia de prácticas.

- El profesorado-tutor de la universidad ha de formar, orientar, atender, acompañar y evaluar al alumnado en prácticas, estar en contacto con los tutores y las tutoras de prácticas y coordinar la experiencia de prácticas y el desarrollo de competencias profesionales del alumnado. En el Practicum II y en el Practicum III se contempla que el tutor o tutora de la universidad realice al menos una visita a los centros escolares.

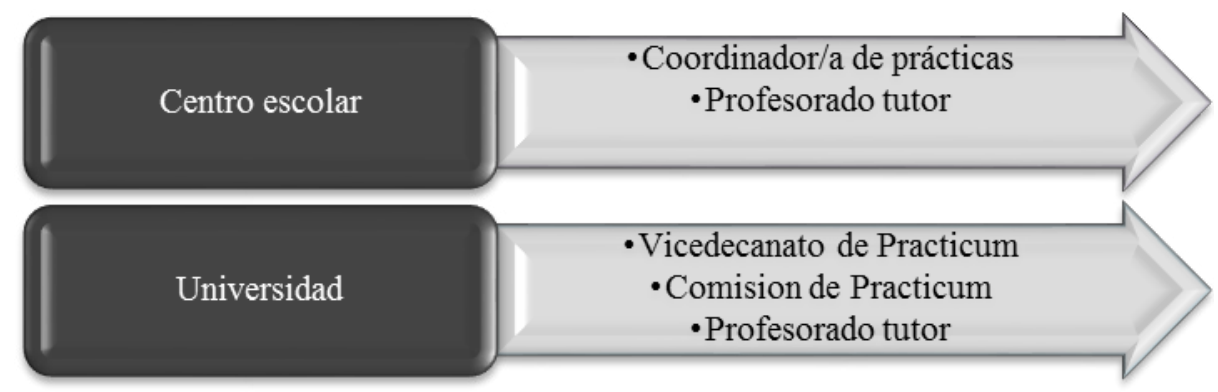

Figura 1. Agentes implicados en las prácticas

Corresponde a los dos últimos agentes mencionados (profesorado-tutor de los centros escolares y profesorado-tutor de la universidad) la formación y evaluación del alumnado en prácticas. Sin embargo, cada agente evalúa diferentes aspectos de las competencias a adquirir por el alumnado y otorga una calificación que tiene diferente peso en la calificación final de la asignatura: el $70 \%$ de la nota final corresponde a la calificación del profesorado-tutor de la universidad y el restante $30 \%$ al profesorado-tutor del centro escolar. Esta evaluación se realiza a través de 
las herramientas diseñadas por la Comisión de Prácticas: la Hoja de Evaluación del tutor o tutora del centro escolar y la Hoja de Evaluación del tutor o de la tutora de la universidad.

En el Practicum I estos indicadores de evaluación se relacionan con los siguientes aspectos competenciales:

1. Conocimiento práctico de la comunidad escolar:

a. Observa y analiza de manera razonada los aspectos organizativos, servicios e instalaciones del centro.

b. Muestra destreza a la hora de observar y valorar el entorno sociocultural y lingüístico del centro escolar.

c. Conoce y refleja los diferentes proyectos educativos que desarrolla el centro.

d. Observa y toma en cuenta los aspectos más significativos sobre la diversidad.

2. Interacción y comunicación:

a. Utiliza la expresión oral y escrita correcta y eficazmente.

b. Mantiene una actitud adecuada ante las distintas interacciones entre docentes y alumnado.

C. Conoce y toma en cuenta los factores de diversidad del alumnado.

3. Variables del aprendizaje:

a. Participa en las actividades: aula, seminarios, reuniones... colaborando con su tutor/a y asumiendo responsabilidades.

b. Es autónomo/a, creativo/a y muestra curiosidad e iniciativa, tomando decisiones cuando la situación lo requiere.

c. Conoce la organización del tiempo, del espacio y su funcionalidad en el aula.

d. Conoce y observa las estrategias metodológicas.

e. Conoce y sabe utilizar los materiales y recursos didácticos del aula y del centro.

f. Identifica y analiza las diferentes capacidades y dificultades que muestra el alumnado durante el proceso de aprendizaje.

Además de estos referentes, la Hoja de Evaluación del tutor o tutora del centro escolar incluye un apartado para que el profesorado-tutor exprese la calificación de la experiencia de prácticas y otro apartado de Observaciones para que pueda realizar las valoraciones que considere oportunas en torno a la evaluación del alumnado. Del mismo modo, esta Hoja de evaluación puede servir de guía al profesorado-tutor de los centros escolares en este período formativo. 
Teniendo en cuenta los aspectos anteriormente descritos, este estudio exploratorio quiere servir de contribución a la reflexión sobre la importancia de la experiencia de prácticas y responder a las siguientes cuestiones:

1. ¿Cómo valoran el alumnado y el profesorado-tutor de los centros escolares la primera experiencia de prácticas?

2. ¿En base a qué criterios valora el profesorado-tutor de los centros de prácticas al alumnado universitario que Ilega a sus aulas?

3. ¿En base a qué criterios construye las valoraciones de su experiencia de prácticas el alumnado universitario?

\section{Metodología}

En este estudio descriptivo y exploratorio se ha considerado el primer período de prácticas (Practicum I) del Grado de Magisterio en Educación Infantil de la Facultad de Educación, Filosofía y Antropología de la Universidad del País Vasco/Euskal Herriko Unibertsitatea (UPV/EHU) en el Campus de Gipuzkoa.

En el curso académico 2016-17 realizaron sus primeras prácticas curriculares en ese período (desde el 17 de octubre al 18 de noviembre de 2016) en 44 centros escolares diferentes, un total de 75 alumnos y alumnas, que fueron supervisados por otros tantos tutores y tutoras de los centros colaboradores y por 3 profesoras-tutoras de la UPV/EHU. A la persona autora de este trabajo le correspondió la tutela del $50 \%$ del alumnado del alumnado de este Practicum I (37 alumnas que realizaron sus prácticas con 36 tutoras y 1 tutor de prácticas de 17 centros escolares públicos o concertados de Gipuzkoa en la etapa de Educación Infantil). El estudio ha recogido y analizado las valoraciones de este grupo de alumnas de la UPV/EHU, así como las valoraciones de sus tutores y tutoras de prácticas (ver Figura 2).

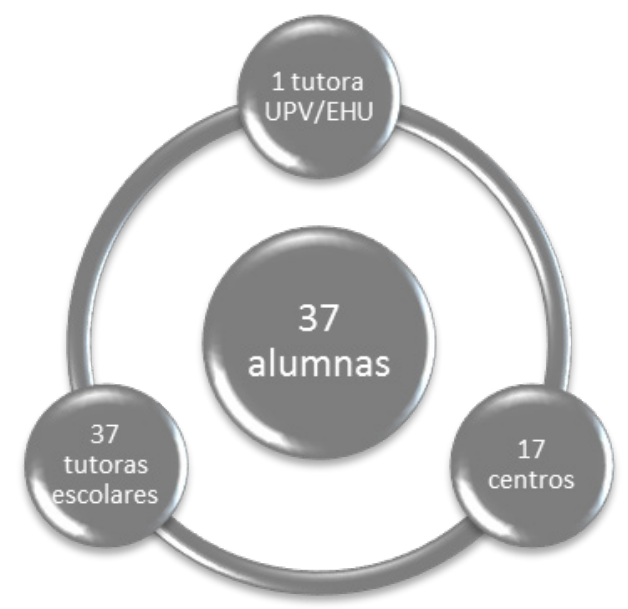

Figura 2. Muestra del estudio 


\section{Procedimiento de recogida de las valoraciones del profesorado-tutor de los centros escolares}

El intercambio de información y la coordinación entre la tutora de la universidad y el profesorado-tutor de los centros escolares se inició la primera semana de la estancia de prácticas. Este contacto necesitó de la colaboración del alumnado en prácticas, a quién en una sesión de tutoría previa a la estancia de prácticas se pidió que, una vez acudiera al centro escolar y se le asignase el tutor o la tutora de prácticas, solicitara a éste/a los datos necesarios para el contacto con la tutora de la universidad (nombre, apellidos, teléfono, correo electrónico y horario). Las alumnas remitieron esta información la primera semana de prácticas y a partir de ese momento el envío de mensajes por correo electrónico fue la vía de comunicación mayoritariamente empleada por la tutora de la universidad para coordinarse con el profesorado-tutor de los centros a lo largo de las cinco semanas de prácticas. En la quinta y última semana de prácticas la tutora de la UPV/EHU solicitó a los/as tutores/as de los centros la cumplimentación y envío de la Hoja de Evaluación del tutor o tutora del centro escolar de las prácticas. Aunque la mayoría de ellos/as $(\mathrm{n}=27$; $73 \%$ de la muestra) lo envió directamente por correo electrónico a la dirección de la tutora de la universidad (como ésta les había solicitado), otra parte importante del profesorado-tutor $(n=10,27 \%)$ de los centros recurrió a vías diferentes a la solicitada (ver Figura 3):

1. La compartieron con la tutora de la universidad por Google Drive $(n=2 ; 5 \%)$.

2. La enviaron a la coordinadora de las prácticas en su centro escolar para que ella la remitiera a la universidad con el resto de los informes de evaluación elaborados por el resto del profesorado-tutor de otro alumnado en prácticas del mismo centro $(n=5 ; 13 \%)$.

3. La enviaron por fax o correo postal a la técnica de la Facultad encargada de la oficina de prácticas $(\mathrm{n}=2 ; 5 \%) ; 0$,

4. Se la dieron directamente a la alumna de prácticas para que fuera ella quien la entregase a la tutora de la universidad $(n=1 ; 3 \%)$.

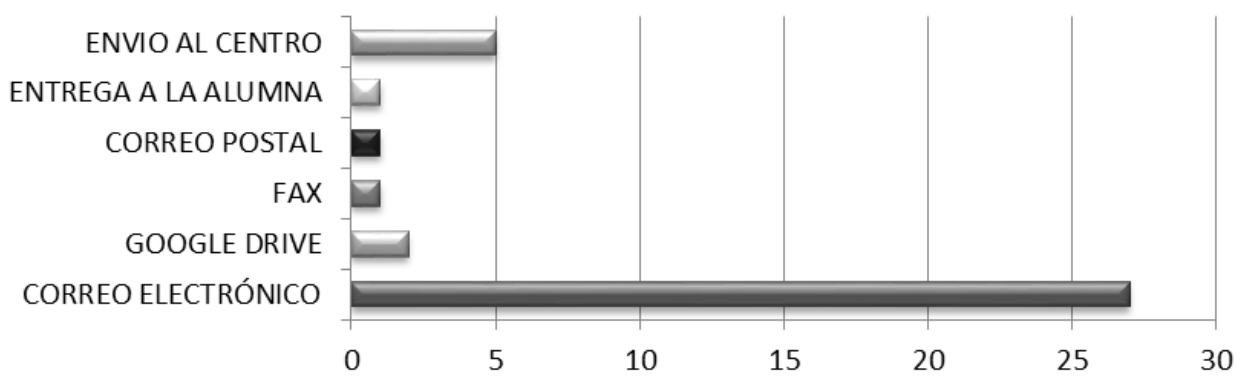

Figura 3. Vías empleadas por el profesorado-tutor para la entrega de la Hoja de Evaluación (\%) 
Como se ha indicado anteriormente, esta Hoja de Evaluación, diseñada por la Comisión de Prácticas recoge un listado de resultados de aprendizaje relacionados con las competencias que el alumnado ha de desarrollar y que el profesorado-tutor de los centros escolares ha de valorar (otorgándoles una puntuación en una escala de 1 a 10). Además, incluye un último apartado para que el profesorado-tutor consigne la calificación numérica final de las prácticas (escala de 1 a 10) y un espacio abierto para aportar sus observaciones y valoraciones. Para este estudio se han recogido y analizado estas calificaciones numéricas y las valoraciones cualitativas del apartado final de la Hoja de Evaluación.

\section{Procedimiento de recogida de las valoraciones de las alumnas}

Las alumnas del Practicum I acudieron a dos seminarios grupales con la tutora de la UPV/EHU: en el primero, previo a la estancia de prácticas, para recibir información y formación sobre las prácticas formativas; en el último, tras la finalización de las prácticas, para valorar la experiencia y recibir información sobre la Memoria Final de Prácticas que debían realizar. En el período de intermedio las tutorías se realizaron a través de una plataforma virtual de apoyo a la docencia. En el seminario realizado a la finalización de las prácticas, la profesora-tutora de la universidad solicitó a las alumnas que valorasen su primera experiencia de prácticas: en primer lugar, calificando la experiencia con una puntuación numérica y, en segundo lugar, empleando un único adjetivo calificativo cuya elección debían argumentar. Estas calificaciones cuantitativas y cualitativas fueron recogidas y contrastadas con las emitidas por el profesoradotutor de los centros escolares.

\section{Resultados}

Se presentan a continuación los resultados de este estudio descriptivo y exploratorio. En primer lugar, se exponen y comparan los datos cuantitativos obtenidos: las calificaciones otorgadas a la primera experiencia de prácticas por el profesoradotutor de los centros escolares colaboradores a través de la Hoja de evaluación y las calificaciones esperadas expresadas por las alumnas. En segundo lugar, se detallan y contrastan los datos cualitativos recogidos: las valoraciones formuladas por el profesorado-tutor y el alumnado.

\section{Calificaciones de la primera experiencia de prácticas}

En general, tanto las calificaciones realizadas por el profesorado-tutor como las esperadas por las alumnas podrían considerarse elevadas.

La puntuación promedio de las calificaciones generales otorgadas por el profesorado-tutor de los centros escolares (ver Figura 4) fue de Sobresaliente (media= 9.6). La mayoría de las alumnas ( $\mathrm{n}=32 ; 86.5 \%$ ) obtuvo una calificación igual o superior a "9" (Sobresaliente). Una única alumna obtuvo la calificación de Aprobado (calificación=6.5). 


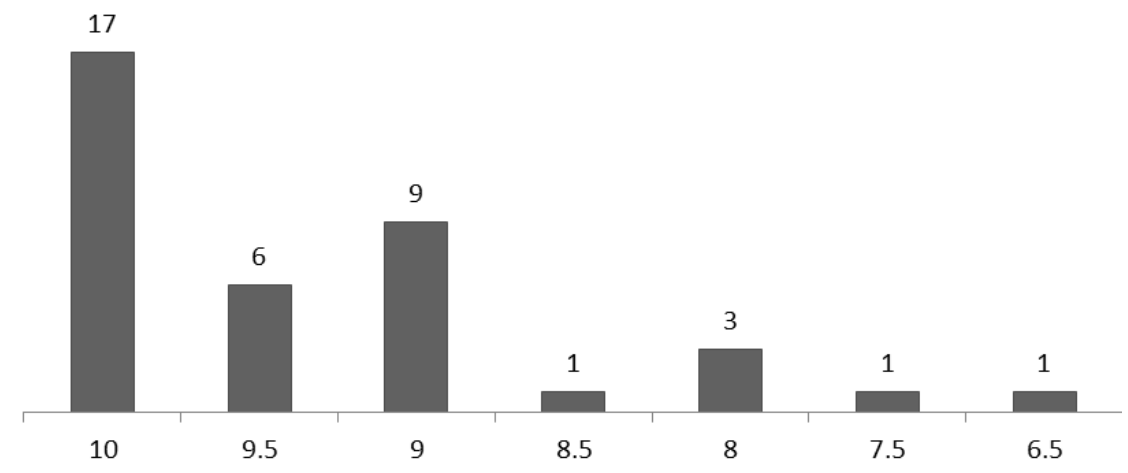

Figura 4. Calificación expresada por el profesorado-tutor en la Hoja de Evaluación final (frecuencia)

Las puntuaciones esperadas por las alumnas (ver Figura 5) también pueden considerarse elevadas, puesto que la puntuación media es menor que la del profesoradotutor y se corresponde con la calificación de Notable (8.7). No obstante, una de cada dos alumnas ( $\mathrm{n}=16 ; 51.6 \%$ ) esperaba una calificación igual o superior a "9" (Sobresaliente) y ninguna manifestó esperar una calificación inferior a "7.5" (Notable).

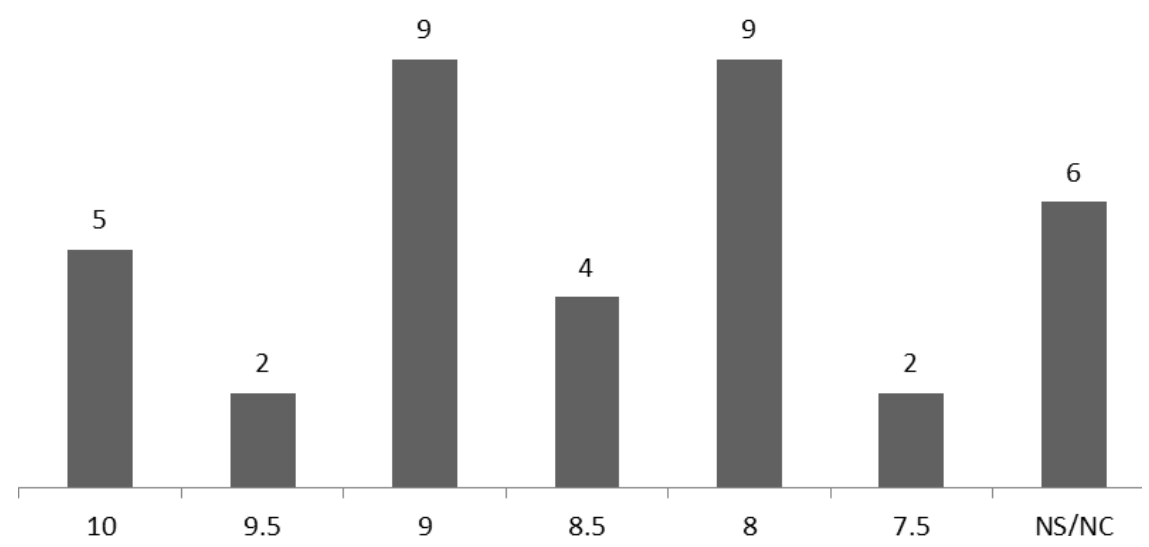

Figura 5. Calificación esperada por las alumnas (frecuencia)

Al comparar las calificaciones realizadas por el profesorado-tutor con las esperadas por las alumnas $(n=31)$ podría por afirmarse que aquellas superaron las expectativas de estas últimas: un $12.9 \%(\mathrm{n}=4)$ de las alumnas formuló una nota esperada entre "1.0" y "1.5" puntos superior a la que obtuvo; un 25.8\% ( $\mathrm{n}=8)$ obtuvo una calificación superior entre "1.0" y "1.5" puntos a la esperada; y un $29 \%$ (n=9) obtuvo una calificación entre "1.5" y "2.5" puntos por encima de la esperada. Puede obser- 
varse la distribución de estas diferencias encontradas entre la puntuación recibida y la esperada en la Figura 6.

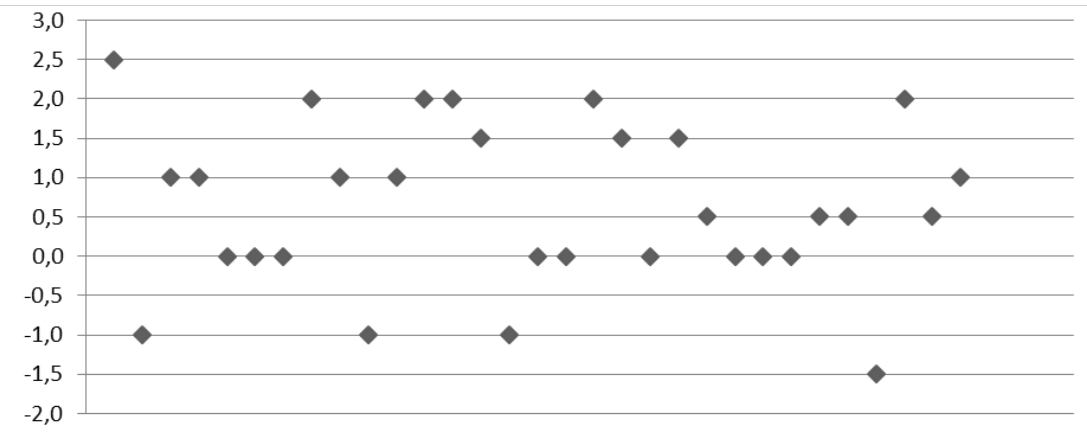

Figura 6. Distribución de las diferencias entre la puntuación esperada y la obtenida por las alumnas

\section{Valoraciones del profesorado-tutor de los centros escolares y de las alumnas}

Como se ha indicado anteriormente, la Hoja de Evaluación de las prácticas externas curriculares que el profesorado-tutor de los centros escolares ha de remitir al profesorado-tutor de la universidad incluye un apartado de respuesta abierta (Observaciones) que fue cumplimentado por 32 de las 37 tutoras (86\%). A continuación se describen los criterios que formularon al argumentar su calificación:

Uno/a de cada dos tutores/as $(\mathrm{n}=16 ; 50 \%)$ destacaron el interés, la actitud adecuada, positiva y/o participativa de la alumna. Aspectos que se corresponden con el indicador $b$ del apartado 2. Interacción y comunicación y con los indicadores a y $b$ del apartado 3. Variables del aprendizaje de la Hoja de Evaluación.

Uno/a de cada tres tutores/as $(n=10 ; 31.2 \%)$ proporcionaron información sobre las tareas realizadas por la alumna y propusieron aspectos a mejorar: "La alumna ha participado en las dinámicas de clase y en las rutinas diarias. Sin embargo, le he tenido que animar a participar en algunas actividades". Otros/as destacaron las capacidades de la alumna de prácticas: "Ha demostrado que tiene experiencia trabajando con niños/as. Además de mostrar iniciativa, ha sido capaz de identificar las necesidades y se ha mostrado creativa y autónoma". Es decir, relacionaron sus valoraciones con el indicador $b$ del apartado 2. Interacción y comunicación y con los indicadores a y $b$ del apartado 3. Variables del aprendizaje de la Hoja de Evaluación.

Un/a de cada cuatro tutores/as ( $n=6 ; 18.75 \%$ ) describieron el proceso de tutoría realizado con la alumna: "Al principio, me dediqué a observar cómo se adaptaba a la dinámica de clase y le vi con poca iniciativa, aunque pensé que era tímida. Hablé con ella y, poco a poco, fue ganando confianza y se ha esforzado cada vez más y los niños y niñas le han cogido cariño. En la próxima estancia tendrá que mostrarse más activa y autónoma". 
Destacaron cómo han vivido ellos mismos o ellas mismas la experiencia de ser tutoras de prácticas: "Ha sido mi primera experiencia como tutora y ciertamente ha sido muy positiva, ya que me ha sido de gran ayuda y, en muchas ocasiones, he tenido la sensación de que tenía una compañera", "Es un grupo-clase complicado y en muchas ocasiones he agradecido la ayuda de la alumna ya que gracias a ella he podido llegar a aplicar estrategias diferentes", "En mi aula entran muchas personas (profesorado de apoyo del centro y especialistas de servicios de apoyo externos) y la alumna me ha sido de mucha ayuda haciéndose en ocasiones cargo de la clase y, especialmente, de una alumna con dificultades.". En los ejemplos descritos puede observarse que aparecen como indicadores las referencias a la ayuda y a la iniciativa del alumnado.

Como se ha señalado en el apartado de metodología en relación al procedimiento de este estudio exploratorio, con el fin de contrastar las valoraciones del profesoradotutor con las valoraciones de las alumnas, en el último seminario de prácticas, la tutora de la universidad les solicitó que describieran su primera experiencia de prácticas calificándola con un único adjetivo.

El 48.4\% de las alumnas (15 de las 31 respuestas obtenidas) valoró su primera experiencia de prácticas como "enriquecedora" ("aberasgarria"). La mayor parte de las restantes valoraciones empleadas por las alumnas pueden considerarse positivas (ver Figura 7): "excelente", "satisfactoria", "maravillosa", "perfecta", "significativa", "útil", "real". Solo una alumna valoró la experiencia como agridulce ("gezigozoa") porque creía que se había mostrado dispuesta a colaborar, pero no había tenido ocasión de aportar algo, para que su tutora la pudiera valorar (fue la alumna que obtuvo la calificación más baja y la tutora le había informado de la misma).

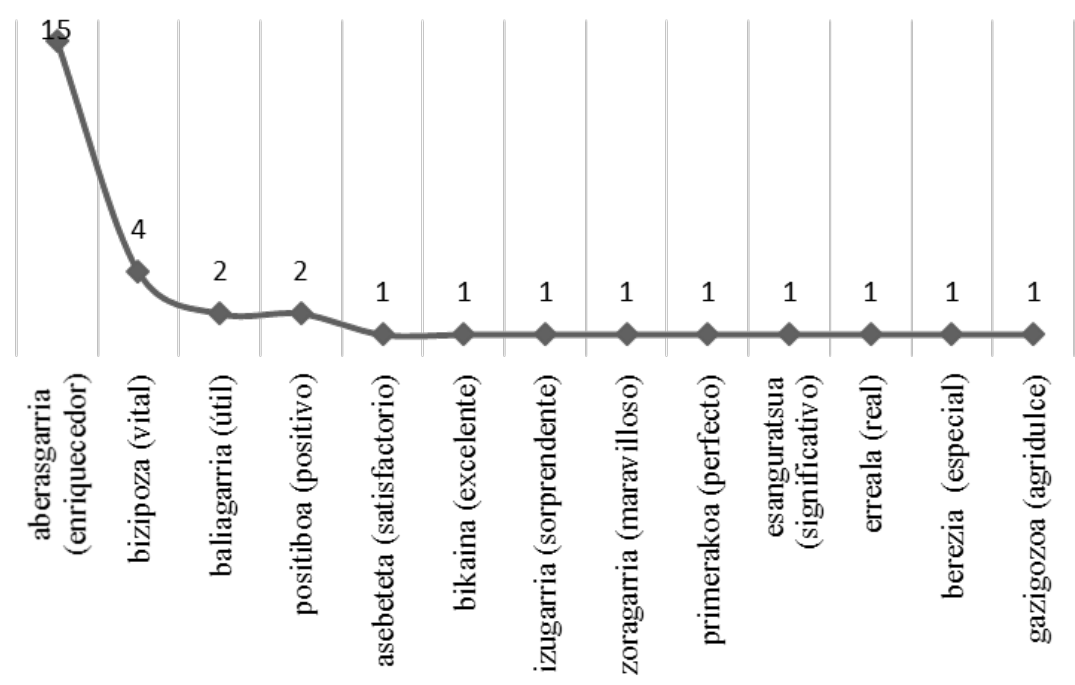

Figura 7. Valoraciones del período formativo expresadas por las alumnas (frecuencia) 
Por otra parte, como se ha indicado anteriormente, estas valoraciones positivas se argumentaron en base a diferentes atribuciones que muestran los criterios empleados como referencia por las alumnas para valorar la experiencia inicial de prácticas (ver Figura 8):

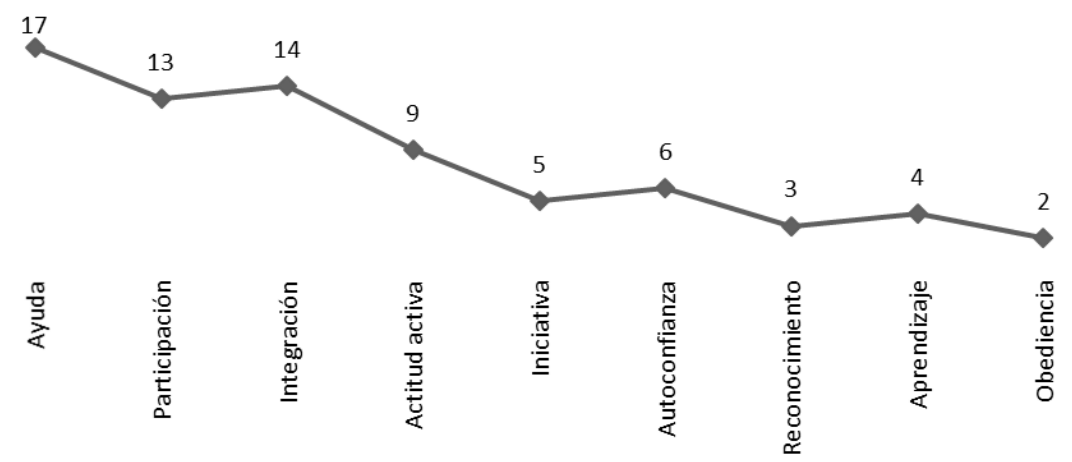

Figura 8. Criterios en los que el alumnado basó su valoración de las prácticas (frecuencia)

En primer lugar, destaca que más de la mitad de las alumnas ( $n=17 ; 54.8 \%$ ) argumentaran su satisfacción con el Practicum relacionándola con la ayuda proporcionada: He ayudado. / He ayudado a la tutora en todo lo que he podido. / He intentado ayudar. / He sido de gran ayuda. / He estado dispuesta a ayudar. / He ayudado en todo lo que he podido. / He ayudado todo lo que he podido. / He ayudado en todo lo que he podido. / He ayudado cuando se necesita ayuda de la mejor manera posible. / He intentado ayudar en todo momento. / He ayudado mucho. / Siempre he estado dispuesta a ayudar, especialmente en un caso especial. / He ofrecido mi ayuda en todo momento. / Porque he trabajado, ofreciendo ayuda en todo momento. / Le he ayudado a la tutora en todo lo que he podido. / He tenido ocasión de hacer mucho trabajo en el aula. / He hecho un gran esfuerzo.

Un $45.2 \%$ de las alumnas $(n=14)$ relacionó esta experiencia satisfactoria con su primera estancia de prácticas con su integración en el centro escolar o en el aula y su grupo social (profesorado, alumnado): Poco a poco me he convertido en una más de la clase. / Me he integrado en la escuela. / Creo que me he adaptado bien. / Creo que me he desenvuelto bien. / Me he adaptado bien a los niños. / He ido creando un vínculo con los niños. / He tratado de buscar el bienestar de los niños. / La actitud de los niños hacia mí sido lo mejor. / He estado a gusto con los niños. / Todos/as (las profesoras, los/as niños/as y yo) hemos estado muy a gusto trabajando. / Los/as niños/as han estado a gusto conmigo y yo con ellos/ as. / Me las he arreglado muy bien con los/as niños/as. / He estado a gusto y me han dicho que han estado a gusto conmigo. / La tutora me ha dicho que han estado a gusto conmigo.

Casi una de cada tres alumnas $(n=13,41.9 \%)$ relacionó su satisfacción con las prácticas con su participación en las mismas $(n=13)$ : He participado. / He tomado parte. / He participado en todo en la medida de mis posibilidades. / Mi participación 
ha sido grande. / Mi participación ha sido muy grande. / He participado haciendo propuestas, preparando fichas, carpetas... / Mi participación ha sido muy activa en todo momento. / He participado activamente. / Por mi participación, aunque creo que podría haber hecho más. / Creo que en todo momento he participado con la tutora y los/as niños/as. / Mi participación ha sido activa. / He participado en las actividades de aula. / He tomado parte activa.

Un 29\% (n=9) del alumnado relacionó su experiencia satisfactoria con su propia actitud activa: He sido activa a lo largo de todo el Practicum. / Siempre he estado dispuesta a hacer lo que había que hacer, a dar mi opinión y a hacer aportaciones. I He puesto interés. / He demostrado interés. / Porque he intentado hacer todo lo que estaba en mis manos. / He estado dispuesta a acudir a las reuniones. / Siempre he estado dispuesta a ayudar. / He trabajado con ganas y con ilusión. / He tenido una actitud colaborativa.

Una de cada seis atribuyó esta valoración positiva a su grado de iniciativa $(n=5$; 16.2\%): He propuesto cosas. / En todo momento he ofrecido ayuda. / He hecho muchas propuestas. / He preguntado lo que no sabía. / Hacía cosas por mi cuenta y sin preguntar a la tutora. También una de cada seis mostró autoconfianza $(n=6 ; 19.4 \%)$ : Me he visto bien en aula. / He hecho un buen trabajo. / Creo que he realizado una buena observación (activa). I Cuando estaba con los alumnos me veía capacitada y pensaba que lo estaba haciendo bien. I Le he trasladado a la tutora nuevas ideas educativas y hemos intercambiado información. I No sé si ponerme un 10 porque creo que la perfección no existe, pero he trabajado bien.

Una de cada ocho alumnas ( $\mathrm{n}=4 ; 12.9 \%)$ argumentó su valoración positiva teniendo en cuenta el aprendizaje realizado: Continuamente he estado aprendiendo y aplicando cosas nuevas. / He aprendido mucho. / He aprendido mucho. / Con esta experiencia he aprendido mucho. Alguna alumna se refiere a la obediencia $(\mathrm{n}=2$; 6.5\%): He hecho lo que la tutora me pedía. / He trabajado siguiendo los principios de la profesora y del centro. Y, una de cada diez consideró el reconocimiento externo recibido $(n=3 ; 9.7 \%)$ : He visto durante todas las prácticas que la tutora confiaba en mí. / Porque mi tutora me dijo que he cumplido bien mis funciones. / La tutora me ha ayudado mucho.

Finalmente, y a pesar de que su valoración general de esta primera experiencia de prácticas había sido positiva, un tercio de las alumnas $(n=11 ; 35.5 \%)$ realizó cierta auto-crítica con sus valoraciones: Al principio, estaba perdida. / Al principio, estaba nerviosa observando todo y preguntando. / Al principio, no me atrevía a participar mucho, pero poco a poco creo que he ido mejorando. / Creo que podría haber propuesto más cosas. / No he demostrado todo lo que podía aportar. / No sé si la tutora esperaba que participara más. / Siempre hay opción de aprender y todavía me queda camino para aprender. / En algunas ocasiones, no sabía cómo actuar y todavía tengo mucho que aprender. / Ha sido la primera experiencia y necesitaría más tiempo. / Es mi primer Practicum y todavía me queda mucho por aprender/ Siempre hay algo a mejorar (me gustaría saber el qué...). 


\section{Discusión}

En primer lugar, en base a los resultados que acabamos de describir y coincidiendo con las investigaciones realizadas por diferentes autores (Cano et al., 2012; Cid y Ocampo, 2007; García et al., 2011; González y Hevia, 2011; Liesa et al., 2009; Manso y Martín, 2014; Martínez y Raposo, 2011a, 2011b; Melgarejo et al., 2014; Pérez y Burguera, 2011; Pontes y Serrano, 2010; Sánchez et al., 2011; Sanjuán y Méndez, 2011; Tello, 2007) podría afirmarse que tanto el profesorado-tutor de los centros escolares de prácticas de Gipuzkoa, como el alumnado de prácticas de nuestro Grado en Educación Infantil valora positivamente esta primera experiencia de prácticas.

En segundo lugar, parece ser que esta satisfacción con las prácticas es mayor en el profesorado-tutor, si consideramos que sus calificaciones fueron más elevadas que las esperadas por el propio alumnado. No obstante, este dato podría ser debido a la falta de experiencia previa del alumnado, que carece de las referencias de otras experiencias de prácticas y que, desconoce las puntuaciones emitidas por su tutor o tutora del centro escolar.

En tercer lugar, las valoraciones positivas realizadas por ambos protagonistas tienen en cuenta factores relacionales e interpersonales como: la ayuda prestada por el alumnado, la iniciativa demostrada, su participación activa y su actitud colaborativa. Por otra parte, en el caso del alumnado, la sensación de haberse sentido integrado en el grupo-aula, tanto por el profesorado-tutor como por los niños y las niñas de la etapa, es un aspecto que parece contribuir a la valoración positiva de sus primeras prácticas externas.

Finalmente, habría que tener en cuenta que las conclusiones de este modesto estudio exploratorio no pueden ser generalizadas, puesto que la experiencia ha sido realizada con una muestra muy reducida de profesorado-tutor y alumnado y en un contexto concreto. Por tanto, sería conveniente ampliar el estudio considerando las calificaciones emitidas por el profesorado-tutor y alumnado de otros cursos del Grado de Educación Infantil para contrastar si las valoraciones de la experiencia de prácticas se mantienen a lo largo de los distintos periodos de prácticas o si, por el contrario, se modifican. En este sentido, sería interesante ampliar la muestra considerando las prácticas de otros Grados universitarios y/o de otras universidades o Comunidades Autónomas.

\section{REFERENCIAS BIBLIOGRÁFICAS}

Cano, J., Orejudo, S. y Cortés, A. (2012). La formación inicial del profesorado de Secundaria: primera investigación en el desarrollo del Practicum de la Universidad de Zaragoza. REIFOP, 15(3), 121-132. Recuperado de http://m.aufop.com/aufop/ uploaded_files/articulos/1364000869.pdf.

Cid, A. y Ocampo, I. (2007). Funciones tutoriales en el Practicum en Psicopedagogía en la Universidad de Vigo: percepción de los estudiantes actuales. Revista de Educación, 344, 285-307. Recuperado de http://www.revistaeducacion.mec.es/ re354/re354_07.pdf. 
Cid, A., Pérez, A. y Sarmiento, J. A. (2011). La tutoría en el Practicum. Revisión de la literatura. Revista de Educación, 354, 127-154. Recuperado de http://www.revistaeducacion. educacion.es/re354/re354_06.pdf.

Elwes Aguilar, O., Fernández Muñoz, E. y Rivas Rebaque, B. (2012). Propuestas para un nuevo modelo de Practicum en la Facultad de Educación de Toledo. REDU. Revista de Docencia Universitaria, 10(3), 441-457. Recuperado de https://dialnet. unirioja.es/servlet/articulo?codigo $=4132312$.

García, E. (2006). Prácticas externas. En M. de Miguel (Coord.), Metodologías de enseñanza-aprendizaje para el desarrollo de competencias (pp. 103-127). Madrid: Alianza.

García, M. S., Pascual, M. A. y Fombona, J. (2011). Las Competencias en el Practicum del Máster en formación del Profesorado de Educación Secundaria Obligatoria, Bachillerato y Formación Profesional. Magister, 24, 109-119. Recuperado de http://hdl.handle.net/11162/4147.

González, M. (2011). El Practicum en la formación docente: mitos, discursos y realidades. En S. Ramírez, C. A. Sánchez, A. García y M. J. Latorre (Coords.), El Practicum en Educación Infantil, Primaria y Máster de Secundaria (pp. 13-29). Madrid: EOS.

González, X. A. y Hevia, I. (2011). El Practicum de la Licenciatura de Pedagogía: estudio empírico desde la perspectiva del alumnado. Revista de Educación, 354, 209-236.

Khortagen, F., Loughran, J. y Russell, T. (2006). Developing fundamental principles for teacher education programs and practices. Teaching and Teacher Education, 22, 1020-1041.

Liesa, M., Otal, P. y Antonio, J. (2009). Valoración de los profesores y alumnos en relación al Practicum en el Grado de Magisterio. En M. Raposo, E. Martinez, L. Lodeiro, J. C. Fernández y A. Pérez (Coords.), El Practicum más allá del empleo: Formación vs. Training (pp.803-824). Actas del X Simposium Internacional sobre Practicum y prácticas en empresas en la formación universitaria, Poio (Pontevedra).

Manso, J. y Martín, E. (2014). Valoración del Máster de Formación de Profesorado de Educación Secundaria: estudio de casos en dos universidades. Revista de Educación, 364, 145-169. http://doi.org/10.4438/1988-592XRE-2014-364-258.

Martínez, E. y Raposo, M. (2011a). Funciones generales de la tutoría en el Practicum: entre la realidad y el deseo en el desempeño de la acción tutorial. Revista de Educación, 354, 155-181. Recuperado de http://www.revistaeducacion.mec.es/ re354/re354_07.pdf.

Martínez, E. y Raposo, M. (2011b). Modelo tutorial implícito en el Practicum: una aproximación desde la óptica de los tutores. REDU. Revista de Docencia Universitaria, 9(2), 97-118. https://doi.org/10.4995/redu.2011.6163. 
Melgarejo, J., Pantoja, A. y Latorre, P. A. (2014). Análisis de la calidad del Practicum en los estudios de Magisterio desde la perspectiva del alumnado. Aula de encuentro, 16(1), 53-70.

Novella, A. (2011). Practicum en re-construcción: Tejiendo colectivamente un modelo de prácticas desde la innovación. REDU: Revista de Docencia Universitaria, 9(3), 259-280. Recuperado de https://dialnet.unirioja.es/descarga/articulo/4019481.pdf.

Ong'ondo, C. O. y Jwan, J. O. (2009). Research on student teacher learning, collaboration and supervision during the Practicum: A literature review. Educational Research and Review, 4(11), 515-524.

Pérez, M. H. y Burguera, J. L. (2011). La Evaluación del Practicum de Pedagogía en el proceso de transición de la Licenciatura al Grado. REDU. Revista de Docencia Universitaria, 9(2), 71-96. https://doi.org/10.4995/redu.2011.6161.

Pontes, A. y Serrano, R. (2010). La formación inicial en un contexto de cambio. En I. González. El nuevo profesor de Secundaria. La formación inical docente en el marco del Espacio Europeo de Educación Superior (pp. 151-165). Barcelona: Graò.

Rodríguez-Gómez, D., Armengol, C. y Meneses, J. (2017). La adquisición de las competencias profesionales a través de las prácticas curriculares de la formación inicial de maestros. Revista de Educación, 376, 229-250. http://doi.org/10.4438/1988592X-RE-2017-376-350.

Rosales, C. (2013). Competencias específicas curriculares que ha de adquirir el estudiante del título de grado de maestro. Profesorado. Revista de currículum y formación del profesorado, 17(3), 73-90. Recuperado de http://www.redalyc.org/ articulo.oa? id=56729527005.

Sánchez, C. A., García, A. y Ramírez. S. (2011). La mejora del Practicum a través de la implicación y reflexión de sus agentes: reflejos de un proceso de investigación-acción. En S. Ramírez, C. A. Sánchez, A. García y M. J. Latorre (Coords.), El Practicum en Educación Infantil. Primaria y Máster de Secundaria (pp. 241-278). Madrid: EOS.

Sanjuán, M. M. y Méndez. M. J. (2011). La valoración y el nivel de satisfacción del alumnado de la USC en sus prácticas escolares. En M. Raposo, M. Martínez, P. C. Muñoz, A. Pérez y J.C. Otero. Evaluación y supervisión del Practicum: el compromiso con la calidad de las prácticas (pp. 1519-1530). Santiago de Compostela: Andavira.

Smith, K. y Hodson, E. (2010). Theorising practice in initial teacher education. Journal of Education for Teaching, 36(3), 259-275.

Tejada, J. y Ruiz, C. (2013). Significación del Practicum en la adquisición de competencias profesionales que permiten la transferencia de conocimiento a ámbitos propios de la acción docente. Profesorado. Revista de currículum y formación del profesorado, 17(3), 91-110. Recuperado de http://www.redalyc.org/articulo. oa?id=56729527006. 
Tello, J. (2007). Implicaciones formativas del Practicum de Psicopedagogía en los centros de Educación Secundaria. Profesorado. Revista de currículum y formación del profesorado, 11, 1-16. Recuperado de https://recyt.fecyt.es/index.php/profesorado/ article/view/41848/23874.

Vick, M. (2006). It's a difficult matter: Historical perspectives on the enduring problem of the Practicum in teacher preparation. Asia-Pacific Journal of Teacher Education, 2(34), 181-198.

Zabalza, M. A. (2011). El Practicum en la formación universitaria: estado de la cuestión. Revista de Educación, 354, 21-43. Recuperado de http://www.revistaeducacion. mec.es/re354_02.html.

Zeichner, K. (2010a). Nuevas epistemologías en formación del profesorado. Repensando las conexiones entre las asignaturas del campus y las experiencias de prácticas en la formación del profesorado en la universidad. Revista Interuniversitaria de Formación del Profesorado, 24(2), 123-149. Recuperado de http://www.redalyc.org/ articulo.oa?id=27419198007.

Zeichner, K. (2010b). Rethinking the connections between campus courses and field experiences in college and university-based teacher education. Educaçao, 3(35), 479-501. 